\title{
Therapeutic Strategies of Stem Cell Transplantation for Liver Cirrhosis
}

\author{
Fan Chen \\ Department of Gastroenterology, Fuzhou General Hospital, Fuzhou, China \\ Email: yangqh4848@sina.com
}

How to cite this paper: Chen, F. (2017) Therapeutic Strategies of Stem Cell Transplantation for Liver Cirrhosis. Yangtze Medicine, 1, 77-95.

https://doi.org/10.4236/ym.2017.12008

Received: March 15, 2017

Accepted: June 18, 2017

Published: June 21, 2017

Copyright $\odot 2017$ by author and Scientific Research Publishing Inc. This work is licensed under the Creative Commons Attribution International License (CC BY 4.0).

http://creativecommons.org/licenses/by/4.0/

\begin{abstract}
Liver transplantation is widely regarded as the most effective therapy for end-stage liver diseases. However, stem cell-based therapy is being developed as a promising strategy which offers a number of benefits as it is minimally invasive and associated with low immunogenicity and low cost. This paper will review the major clinical issues surrounding the use of stem cell therapy for managing cirrhosis, such as discussing the selection of appropriate subtypes of bone marrow stem cells and the need for pre-differentiation into hepatocyte-like cells prior to transplantation, and providing an overview of the methods to improve cell viability and to prevent the exacerbation of cirrhosis. The role of human umbilical cord blood stem cells and amniotic epithelial cells for the treatment of liver disease will be also introduced.
\end{abstract}

\section{Keywords}

Liver Cirrhosis, Stem Cell Therapy

\section{Introduction}

Cirrhosis is a common consequence of chronic liver diseases, which leads to the liver failure during the decompensation stage. Liver transplantation is the only therapeutic option for advanced cirrhosis. However, the practicality of liver transplantation is compromised by the severe shortage of donated organs, high medical costs, and long-term requirement for immunosuppression. Sources of hepatocytes for the bio-artificial liver and hepatocyte transplantation are also limited. Recent studies suggest that stem cell transplantation may be a promising new strategy for the treatment of end-stage liver diseases [1] [2]. This treatment has not been widely used currently because of various unknown risks. And the treatment is expected to be gradually mature and standardized through the efforts of scholars. 
Here we review therapy-related details on the use of stem cell treatment for liver cirrhosis, and discuss advances in human umbilical cord blood stem cells (UCBSCs) and human amniotic epithelial cells (hAECs) as sources of therapeutic stem cell.

\section{Selection of Bone Marrow Stem Cells for Liver Cirrhosis}

\subsection{Hematopoietic Stem Cells or Mesenchymal Stem Cells?}

\subsubsection{Clinical Studies}

It is widely accepted that both liver injury and hepatic fibrosis are closely related to the immune response. Hematopoietic stem cells (HSCs) including CD34 $\mathrm{CD}_{133^{+}}$and mononuclear cells enhance immune function, whereas, mesenchymal stem cells (MSCs) have an immunosuppressive effect. The type of the stem cells used for managing liver disease, therefore, depends on cellular immune function or the extent of the cellular immune response which varies according to the etiology and stage of liver diseases.

Autoimmune liver diseases are commonly associated with enhanced cellular immune responses, and the use of $\mathrm{CD} 34^{+}$and/ or $\mathrm{CD} 133^{+}$cells exacerbates this condition [3] [4], whereas MSCs provide a safe and effective treatment strategy [5]. While there is no initial evidence of impaired immune response during the early stages of alcoholic liver cirrhosis [6], the immune response may be reduced later as the disease progresses, especially in the advanced stages of liver failure [7]. As a result of this disease profile, HSCs are eliminated by the active immune cells during the treatment of early-stage alcoholic liver cirrhosis, and accompanied by exacerbating the progression of hepatic fibrosis [8]. A previous study shows that two among eight patients receiving HSCs for the treatment of alcoholic liver cirrhosis, there was one case of diabetes (an autoimmune disease) with graft-versus-host disease, and another patient developed deterioration of liver function with the hemorrhage of the upper digestive tract [9]. Jang et al. [10] treated alcoholic cirrhosis with MSCs and found no adverse events.

However, the reduced cellular immune response was usually found in the patients of alcoholic liver cirrhosis complicated with HCV [6], hence the HSCs transplantation did not result in any adverse effect related to the immune response, which has been confirmed by Couto BG [9]. Depressed cellular immune function is often shown in patients with HBV- or HCV-induced cirrhosis [11]. Salama et al. [12] treated HCV infection complicated cirrhosis using HSCs $\left(\mathrm{CD} 34^{+} / \mathrm{CD} 133^{+}\right)$. In these subjects there were no adverse immune responses following HSCs transplantation and HCV titers were reduced in most patients with liver cirrhosis at 6 months post-transplantation. It was therefore, postulated that HSCs treatment may inhibit HBV propagation in patients with HBV- induced cirrhosis [13].

Cellular immune function is generally enhanced during the early stages of cirrhosis complicated by acute-on-chronic liver failure (ACLF), but becomes depressed during the later stages of the disease process [14]. Therefore, appropriate types of stem cells should be selected according to the cellular immune 
status of patients at the beginning of treatment. However, a recent clinical trail has been undertaken by using allogeneic bone marrow mesenchymal stem cells (BMSCs) through peripheral vein in treatment of patients with different phase of HBV ACLF. The results indicated that transplantation of BMSCs only in plateau phase rather than in advanced phase can improve survival rate of the patients [15].

\subsubsection{Summary}

Treatment with MSCs may be suitable for patients with early stage alcoholic liver cirrhosis, and for those with autoimmune liver disease, or early stage of liver cirrhosis complicated with ACLF. The use of HSCs is appropriate for late stage alcoholic liver cirrhosis, and for HBV- or HCV-induced cirrhosis, alcoholic liver cirrhosis complicated with HCV or HBV infection, and late stage of liver cirrhosis complicated with ACLF. (As illustrated in Figure 1) [16].

Adipose-derived mesenchymal stem cells (ASCs) should be used instead of BMSCs for early stage of liver cirrhosis complicated with ACLF. This is because BMSCs have to be cultured and propagated in vitro making the procedure too slow to meet the urgent needs of these patients.

It has been suggested that endothelial progenitor cells (EPCs) might be a source of stem cells for cirrhosis therapy [17]. Since EPCs share a range of biological features with HSCs [18], the therapeutic indications for EPCs might also be similar to those for HSCs.

\subsection{Predifferentiated or Undifferentiated Stem Cells Prior to Transplantation?}

\subsubsection{Laboratory Findings}

At the laboratory level, predifferentiated stem cells show better therapeutic effects than undifferentiated cells for the treatment of liver fibrosis [19] [20] [21]. There might be multiple reasons listed and discussed as follows: First, induction

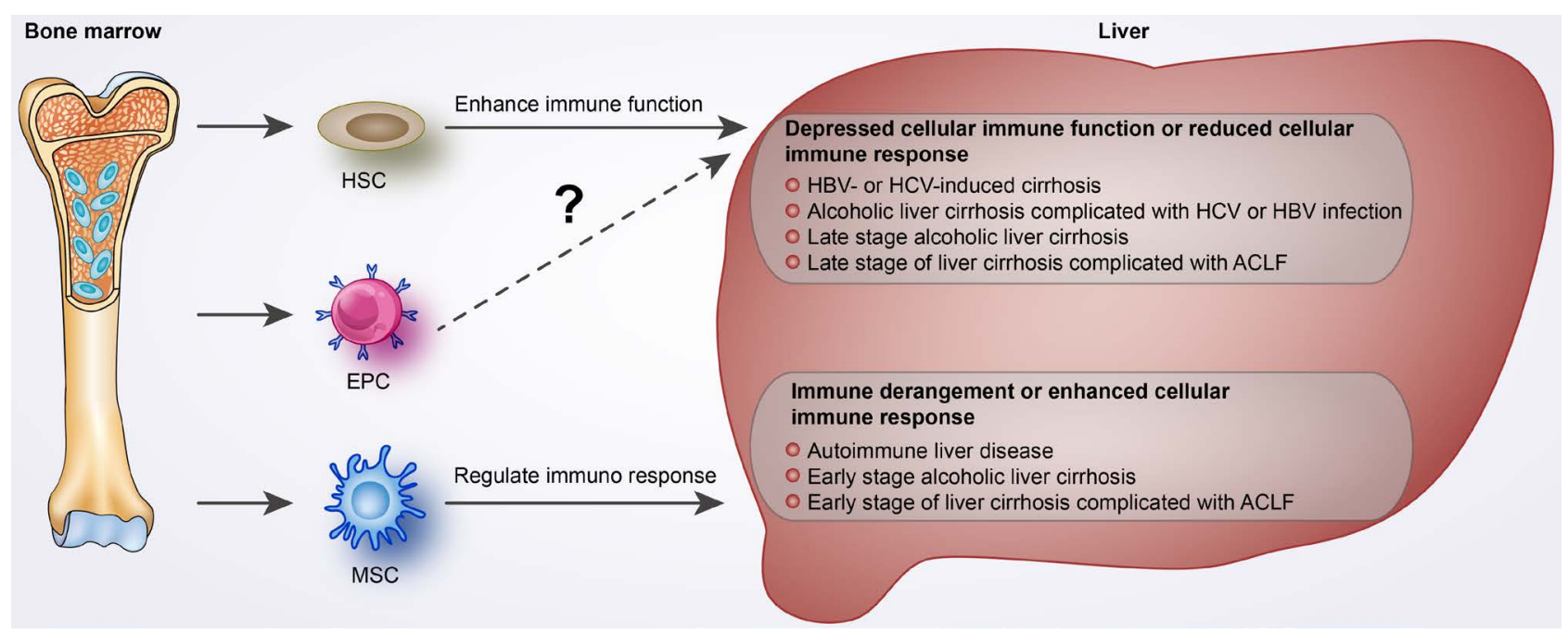

Figure 1. The indications of different bone marrow stem cell types for liver cirrhosis therapy (modified from the figure in reference 16). Abbreviations: HSC: Hematopoietic stem cell; EPC: endothelial progenitor cell; MSC: mesenchymal stem cell; ACLF: acute-on-chronic liver failure. 
before transplantation may facilitate stem cell differentiation into hepatocyte, resulting in increased therapeutic effect [22]. Secondly, it is also possible that the pathological hepatic microenvironment which may include inflammation concurrent with fibrosis or cirrhosis may cause stem cells to differentiate into different types of mature cells [23] [24], such as stellate cells and myofibroblasts. This sequence of events would further promote fibrosis. Stem cell induction before transplantation has been used to prevent the differentiation pluripotency. Thirdly, the unpredifferentiated bone marrow stem cells (BMCs) which are undifferentiated juvenile cells, do not enter the hepatic differentiation process and are unstimulated cells. Therefore, they have limited proliferative ability in the microenvironment of the liver and are unable to active the endogenous hepatic stem cells or progenitor cells. By contrast, the pre-differentiated stem cells have entered into the process of differentiation and become stimulated hepatocytelike cells, thus they have the capacity to propagate rapidly after transplantation, activate endogenous hepatic stem cells and produce a satisfactory therapeutic effect [25].

Other sources of laboratory evidence indicate that undifferentiated stem cells have a better therapeutic effect than predifferentiated stem cells [26] [27] [28]. This is thought to be because the liver regenerating and anti-fibrotic effects of stem cells is the result of their paracrine ability rather than an outcome of directed differentiation. The paracrine capability of predifferentiated stem cells has been shown to be attenuated during in vitro induction [29], though there are different viewpoints [30].

\subsubsection{Clinical Studies}

Comparison of the effects of transplanting predifferentiated and non-predifferentiated BMSCs via peripheral vein for the treatment of HCV-liver cirrhosis indicates that there is no significant difference with respect to clinical outcome and laboratory examinations at 6 months follow-up [31].

\subsubsection{Summary}

However, the individual therapeutic merits of predifferentiated and unpredifferentiated stem cell transplantation for the treatment of cirrhosis remain controversial. Based on the published results, thoroughly predifferentiated stem cells appear to be less potent than unpredifferentiated cells [27] [28], and hepatocyte transplantation appears to be less effective than stem cell transplantation [32]. These findings suggest that partial in vitro induction of stem cells, which actives stem cells and preserves their paracrine capability, is the better treatment option [33].

\section{Enhancing Stem Cell Survival}

The therapeutic effect of stem cell transplantation is seldom satisfactory in late stage cirrhosis, when the microenvironment has been severely damaged. Studies have shown that serum albumin levels (ALB) of cirrhotic patients with ChildPugh stage A or B hepatic impairment, can be significantly improved by stem 
cell transplantation, The mean serum ALB value of transplantation group was $(33.22 \pm 2.85)$ and $(29.65 \pm 4.36) \mathrm{g} / \mathrm{L}$ for cirrhotic patients with Child-Pugh stage $\mathrm{A}$ and $\mathrm{B}$ initially. The value rised to $(36.74 \pm 4.37) \mathrm{g} / \mathrm{L}$ and $(33.31 \pm 5.77) \mathrm{g} / \mathrm{L}$ after 24 weeks, when comparing the value before transplantation, $(P<0.05)$. Whereas, there was no significant improvement in ALB in patients with Child-Pugh stage $C$ cirrhosis. For cirrhotic patients with Child-Pugh C, the mean serum ALB value rised from $(27.97 \pm 3.04)$ to $(29.24 \pm 4.91)$ after 24 weeks, when comparing the value before transplantation $(P>0.05)$ [34] [35]. Based on these findings it was postulated that severe damage of the liver function might be closely related to poor stem cell survival post transplantation.

This was further demonstrated by the ex vivo experiments showing that hepatic-directed differentiation was not apparent in BMSCs induced by the serum from patients with $5 \%$ liver injury. In the same experiments apoptosis occurred in some of stem cells induced by serum from patients with $10 \%$ liver injury and all stem cells died after induction with serum from patients with $15 \%$ liver injury [36]. Although, serum from patients with hepatic injury might be able to facilitate the hepatic differentiation of the stem cells in vitro; toxicity from the damaged liver may impair stem cell viability during in vitro induction.

\subsection{Methods to Improve the Microenvironment}

\subsubsection{Hyperbaric Oxygen Treatment}

Systematic hypoxemia persists in $30 \%$ to $70 \%$ of patients with cirrhosis. Longterm hypoxemia results in apoptosis of transplanted stem cells, and affects therapeutic outcomes [37]. Clinical studies have shown that hyperbaric oxygen improves the hypoxemic condition of the hepatic microcirculation, and decreases toxic metabolites in plasma, thereby improving stem cell survival [38]. However, hyperbaric oxygen treatment may not be suitable for patients with esophageal varices. In these subjects normobaric oxygen treatment can be suggested instead.

\subsubsection{Medication and (or) Plasma Exchange to Improve the Hepatic Microenvironment}

Plasma exchange has been shown to increase serum levels of hepatocyte growth factor (HGF) without changing serum levels of fibroblast growth factors (FGF-4 and bFGF) or epidermal growth factor (EGF). Plasma exchange may, therefore, promote the differentiation of BMCs in a way that allows them to repair and regenerate the impaired hepatocytes in patients with severe hepatitis [39]. Experiments using the pig model of acute liver failure indicate that autologous MSCs transplantation in combination with medication (including $100 \mathrm{ml}$ plasma and $100 \mathrm{mg}$ diammonium glycyrrhizinate intravenously injection) resulted in significantly better outcomes than MSCs transplantation or medication alone [40]. Another clinical trial showed that combined therapy with umbilical cord blood infusion and plasma exchange improved the liver function and immunity to a greater extent than monotherapy in patients with chronic severe hepatitis [41].

Thus, both medication and stem cell transplantation effectively reduce inflammation, improve liver function and prevent further deterioration. These 
procedures allow toxic metabolites to be quickly removed providing a relatively stable and favorable internal environment for stem cell survival and propagation.

\subsubsection{Appropriate Transplantation Site}

The spleen is adjacent to liver, and for this reason intrasplenic transplantation is usually preferred when the hepatic environment is not suitable for stem cell transplantation. Transplantation of induced MSCs into the spleen, portal vein, and vena caudalis was shown to improve liver function and the pathohistological profile of patients with chronic liver disease, irrespective of which route was used. However, intrasplenic transplantation was the most effective of the three routes [42].

\subsection{Stem Cell Engineering}

There are multiple types of stem cell resources for use in treating cirrhosis. These include fetal liver stem cells, embryonic stem cells, UCBSCs, BMCs, amniotic stem cells, and induced pluripotent stem cells [43]. It has long been believed transplantation with a single stem cell type (such as MSCs) might be more effective than using a mixed type (such as a mononuclear cells). However, accumulated evidence suggests that cell-cell interactions may be an important factor for the success of the stem cell therapy, and that cytokines or specific cell types in mixed mononuclear cells, might be associated with improved stem cell survival and therapeutic effect [44]. A previous study reported that co-trans- plantation of two types stem cell might be an optimal method [45]. Results from another clinical study suggest that UCBSCs transplantation might be superior to BMCs [46]. However, further experimental confirmation is need to determination the types of stem cell that are best adapted to survive the harsh environment of liver.

\subsubsection{In Vitro Preconditioning}

Results from a rat model of liver injury, showed that MSCs co-cultured with hepatocyte growth factor for 2 weeks survived better than control cells, and significantly reduced the degree of liver fibrosis [19]. In another experiment, transplantation of microencapsulated MSCs significantly increase the survival rate of $90 \%$ hepatectomized rats. The 2-week survival rate of microencapsulated MSCs group, free MSCs group and 90\% hepatectomized group were $91.6 \%, 25 \%$ and $21.4 \%$ after transplantation, $(P<0.01)$. The microencapsulated MSCs exhibited long-term survival, secreted trophic factor and differentiated into hepatocyte-like cells [47]. In another study [48], interleukin 10 gene-modified bone marrow-derived liver stem cells (BDLSCs) were shown to significantly reduce inflammation associated with liver fibrosis. They also promoted liver regeneration, to a greater degree than unmodified BDLSC.

\subsubsection{Hepatogenic Differentiation of Stem Cells Prior to Transplantation} A model of rat spinal cord injury [49] showed that predifferentiated MSCs had better motor function and a higher survival rate than undifferentiated MSCs. In 
another study, MSCs co-cultured with the hepatocyte growth factor and basic fibroblast growth factor were found to a have superior therapeutic effect to control MSCs in a rat model of liver fibrosis [21].

\subsubsection{Inhibition Stem Cell Apoptosis}

It has been shown that exposure of rat hepatocyte-like cells to ursodeoxycholic acid (UDCA) inhibits deoxycholic acid-induced hepatocyte-like cells apoptosis, by down-regulating the p53/Bax signal pathway [50].

\subsection{Summary}

Stem cell transplantation has been shown to be associated with an unsatisfactory therapeutic effect in the treatment of liver failure especially for subjects with severe Child-Pugh stage $\mathrm{C}$ cirrhosis. One key reason for this is that the transplanted stem cells may not be able to survived the relatively hostile microenvironment of the liver. Promotion of the survival rate of the transplanted stem cells, therefore, appears to be critical for the success of treatment. To achieve this, a suitable transplantation strategy should be considered on basis of the stem cells per se and the hepatic microenvironment (as illustrated in Figure 2).

\section{Prevention of Hepatic Fibrosis}

Accumulated studies suggest that fibrosis can be ameliorated by stem cell transplantation [51] [52]. However, there is equally compelling evidence indicating that stem cells have the potential to differentiate into the hepatic stellate cells and myofibroblasts, both of which are involved in the progression of fibrosis

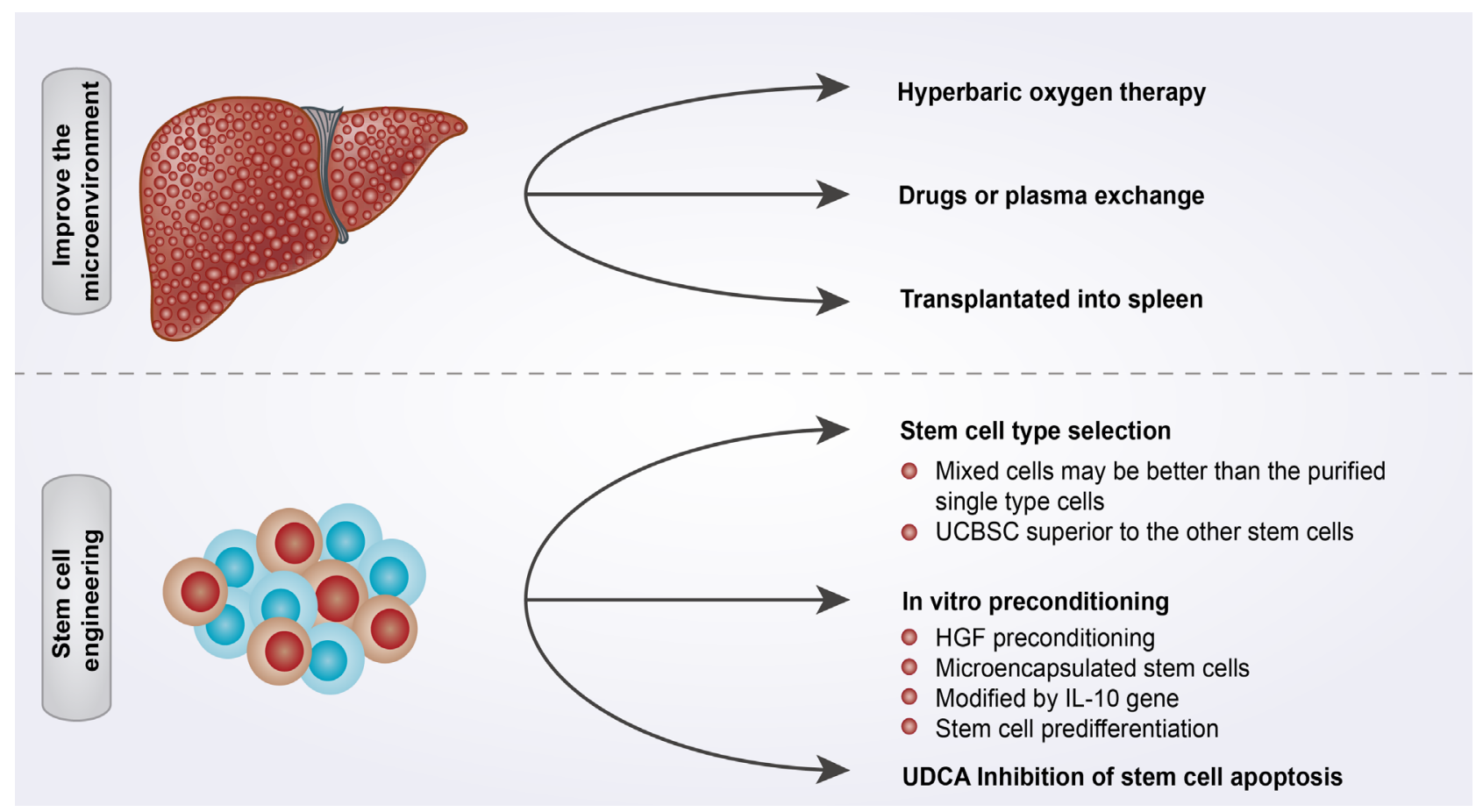

Figure 2. The strategies to improve stem cell survival. Abbreviations: UCBSC: umbilical cord blood stem cells; HGF: hepatocyte growth factor; IL-10: interleukin 10; UDCA: ursodeoxycholic acid. 
[23]. It is therefore, important to address the issue of preventing stem cell induced fibrosis.

\subsection{Etiological Treatment}

Stem cell transplantation is not routinely combined with etiological treatment such as anti-HBV or HCV therapy, and as result liver function is improved, but portal hypertension and upper gastrointestinal hemorrhage are not alleviated [34]. The more recent, combined therapeutic strategy of using stem cell transplantation and etiological treatment has been shown to improve both liver function and upper gastrointestinal hemorrhage [53]. Persisting inflammation in the liver due to lack of etiological treatment not only interferes with the therapeutic effect of transplanted stem cells, but also drives stem cell differentiation towards the production of myofibroblasts and hepatic stellate cells, both of which exacerbate the fibrosis. By contrast, etiological treatment used in conjunction with stem cell transplantation synergistically improves the therapeutic outcome of patients with cirrhosis.

\subsection{Anti-Inflammatory Treatment}

It is generally believed that under the microenvironment of fibrosis or cirrhosis most transplanted stem cells differentiate into hepatocytes, and hepatic stellate cells by hepatic stellate cells [24] [54]. However, in a mouse model of liver fibrosis or cirrhosis with remarkable inflammation [23], the majority of injected stem cells differentiated into myofibroblasts, resulting in liver fibrosis being aggravated. Under such circumstances an appropriate anti-inflammation therapy should be administered to improve the survival of the transplanted stem cells [40], to avoid stem cells differentiation into myofibroblasts, and thereby improve the therapeutic effect. However the anti-inflammatory effect should not be so strong that it impairs stem cell motility and differentiation [55] [56].

\subsection{Selection of an Appropriate Stem Cell Type}

The type of stem cells should be selected cautiously and carefully to avoid enhancing the immune response and triggering an immune system disorder [3] [4] [8] [9]. MSCs is preferred over HSCs for the cirrhosis with the severe inflammation. Whole bone marrow cells or bone marrow mononuclear cells (BMNCs) contain a certain proportion of inflammatory cells and a relatively small proportion of HSCs. Consequently, the resulting anti-inflammatory effect is less than that seen with MSCs. Experiments using a rat model of severe cirrhosis showed that liver fibrosis was exacerbated by BMNCs transplantation, but was ameliorated by BMSCs transplantation [57] [58] [59]. Similarly, it was demonstrated by the animal experiments that BMSCs had better therapeutic effect on acute liver injury with the respects to anti-inflammation and anti-fibrosis activity [60].

\subsection{Summary}

Stem cell transplantation may have either positive or negative outcomes depen- 
ding on the direction of stem cell differentiation which can be uncertain within the complex microenvironment of the liver. A satisfactory therapeutic effect can be achieved by improving the hepatic microenvironment, by selecting appropriate types of stem cell, and by in vitro manipulation prior to transplantation.

The use of stem cell induction prior to transplantation has been described previously and the therapeutic use of hAECs will be described in a following section.

\section{Umbilical Cord Blood Stem Cells}

Umbilical cord blood, has been used for many years in clinical practice as an important source for hematopoietic stem cell transplantation, and has resulted in encouraging therapeutic outcomes. Experimental trials have shown that UCBSCs can be induced and differentiated into hepatocyte-like cells [61]. They stimulate liver regeneration [62], alleviate fibrosis [63], enhance hepatic angiogenesis [64], and inhibit primary hepatocellular carcinoma [65]. They, may, therefore, provide be suitable for use in a wide range of therapeutic applications for end-stage liver disease.

\subsection{Experimental Studies with UCBSCs}

Liver function and hepatic histological grade were significantly improved in rats with decompensated cirrhotic rats after injection through the tail vein of a nucleated cell suspension of umbilical cord blood containing $>2 \times 10^{6} \mathrm{CD} 34^{+}$stem cells [66]. Liver fibrosis and survival rate were also improved relative to control rats although the differences were not statistically significant. In a rabbit model of fibrosis transplantation of $5 \times 10^{5} \mathrm{CD} 34^{+} \mathrm{UCBSC}$ via portal vein injection (in the absence of immunosuppression) improved liver function and alleviated fibrosis without causing symptoms of rejection [67]. Based on these findings, it was proposed that following intrahepatic injection into the rats with liver cirrhosis, umbilical cord blood mononuclear cells (UCBMNCs) are differentiated into hepatocytes and inhibit the activation of stellate cells, which is shown by the induction of stellate cells apoptosis [68]. This form of hepatocyte regeneration, coupled with the anti-inflammatory and antioxidant effects of UCBMNCs, is thought to alleviate cirrhosis. The same studies showed that UCBMNCs induced high levels of expression of the APE1 gene which might also be responsible for the prevention and inhibition of hepatic injury.

However, in a similar experimental setting [69], $1 \times 10^{5}$ transplanted CD $34^{+}$ UCBMNCs failed to become engrafted in the rat liver, possibly as a result of thioacetamide which was used to induce the cirrhosis. In another study injection of $10 \times 10^{6}$ UCBMNCs to the liver via the portal vein, resulted in impairment of liver and kidney function, with no positive pathological evidence of improvements in liver tissues. Based on the findings of this study it was concluded that UCBMNCs transplantation exacerbates the liver injury and induces unwanted hepatorenal syndrome [70]. The adverse effects of stem cell therapy on renal and liver function in this study might have been related to an overdose of trans- 
planted stem cells which resulted in a rejection response. The rejection response between the human UCBSCs and human recipients might be less marked than that between human UCBSCs and rat recipients. However, further clinical trials are needed to more precisely elucidate the effect of UCBSCs.

\subsection{Clinical Studies with UCBSCs}

UCBSCs transplantation via hepatic artery has been undertaken in HBV patients with cirrhosis and Child Pugh stage $C$ liver impairment. These patients were receiving anti-viral agents, HGF, and other symptomatic and supportive treatments. The results indicated significant improvements in symptoms and liver function, and CT examination showed that the liver cross-sectional area was markedly increased compared to pretreatment findings [71]. The satisfactory outcomes reported in this study might in part be explained by the use of associated therapies that addressed the underlying etiology and promoting stem cell survival.

In the another trial [72] UCBSCs were transplanted through the hepatic artery or portal vein in a similar cohort of patients with cirrhosis, HBV and Child Pugh stage $\mathrm{C}$ liver impairment. In this study hepatic function and the clinical symptoms significantly improved without obvious adverse effects or complications. Unfortunately, no comparative data exists that compares portal vein and hepatic artery transplantation.

Transplantation of UCBSCs via the hepatic artery of patients with cirrhosis of various etiologies, with Child Pugh stage B or C hepatic impairment, resulted in a slight decrease in serum ALB during the initial stage of treatment, indicating the need to monitor patients closely post transplantation, though clinical symptoms, liver function, and blood coagulation function improved during the subsequent 6 month follow-up period [73].

UCBSCs transplantation has also been used for the treatment of cirrhosis associated with hepatic diabetes. In this study, improvements in cirrhosis were accompanied by normalization of blood glucose levels [74]. In another study UCBSCs transplantation resulted in a general improvement in the overall condition of a patient with Wilson disease, concomitance with increased appetite for food. Serum ceruloplasmin levels were increased and no acute rejection occurred [75].

A study comparing UCBSCs transplantation with the autologous peripheral blood stem cells (PBSCs) transplantation for treatment of severe chronic liver disease with ascitic cirrhosis, indicated that both types of stem cells improved liver function and cirrhosis with equal effect [76]. However, UCBSC transplantation was considered to be more effective than PBSC in patients with the most severe disease. Another study comparing UCBSC with BMCs transplantation showed that higher proportions of $\mathrm{CD} 34^{+}$or $\mathrm{CD} 38^{+}$cells were contained in UCBSCs than in BMCs. Both stem cell types were used to treat HBV and HCV cirrhosis in patients with Child Pugh stage B and C liver impairment. The results showed that the therapeutic effect of UCBSCs transplantation was better than 
that of BMCs [46].

\subsection{Summary}

Based on published literature, UCBSCs transplantation for the treatment of cirrhosis appears to be superior to PBSCs or BMCs transplantation. Although the immunogenicity of the UCBSC is very low, it still contains allogeneic cells and close monitoring is required to ensure early detection and prevention of any rejection responses.

\section{Amniotic Epithelial Cells and Liver Disease}

Recent studies show that human amniotic epithelial cells (hAECs) might possess certain biological characteristics and functions of hepatocytes [77] [78] [79]. Indeed, it has been shown that hAECs can be induced to differentiate into hepatocyte-like cells and cholangiocytes [80] [81]. The potential advantages of using hAECs include the wide range of available sources and the simple collection method. More than $1 \times 10^{8}$ hAECs can be extracted from a single abandoned placenta amnion [82]. There are no medical ethical issues to address. Undifferentiated cells display low levels of immunogenicity, however, hAECs that have differentiated into hepatocyte-like cells may possess a certain level of immunogenicity [83]. hAECs have also been shown to have antifibrotic properties and anti-inflammatory effects [84], which make them suitable for the treatment of various liver diseases.

\subsection{Genetic Liver Diseases}

Transplantation of $5 \times 10^{5}$ hAECs cells every other week via the tail vein prolonged the survival of mice with experimentally induced Niemaoh-Pick disease [85]. Loss of body weight, organ damage, cholesterol deposition, and the relative weight of the liver were all reduced, indicating that hAECs may have a role in the treatment of fatal genetic diseases, including the metabolic liver disease.

\subsection{Liver Fibrosis}

Amniotic membrane administered as a patch on the surface of the liver has been investigated in a biliary fibrosis model established by bile duct ligation in rats [86]. The amniotic membrane reduced biliary cell inflammation and collagen deposition in the liver. It also inhibited the activity of myofibroblasts, which significantly reduced and delayed the development of liver fibrosis. Based on these findings it was postulated that its therapeutic effect might be due to paracrine activation rather than to the hAECs per se. Other workers have shown that hAECs injection improves liver function, reduces hepatocyte apoptosis, and decreases hepatic inflammation and fibrosis in a CCL4-induced liver fibrosis model in wild-type mice [87]. In this study no obvious rejection response was seen 2 weeks after hAECs transplantation.

These findings suggest that, hAECs may provide an effective treatment for liver fibrosis, as it appears to avoid the exacerbation of fibrosis that is caused by 
stem cell differentiation. The lack of rejection and exacerbation of fibrosis in the two mouse models also supports the use of hAECs as a potential source of stem cells for the treatment of cirrhosis.

\subsection{Summary}

The efficacy and safety of human amniotic membrane in the treatment of ophthalmological diseases have been established for a number of years. However, there remains a lack of in-depth studies using hAECs for liver regeneration in liver disease, and therefore requiring rigorous research.

\section{Conclusion}

Stem cell therapy has been shown to be effective for treating end-stage liver disease. Further investigations using careful study designs should be undertaken to identify the optimal dose of injected stem cells, the times of transplantation, the timing of repeated cell transplantation, and the monitoring on the long-term complications. With the gradual promotion of stem cell therapy, we can focus on the optimization of the treatment methods in the future, especially on the improvement of curative effect and the lasting aspect of therapeutic efficacy. It is believed that the formal clinical application will be realized in the future by the joint efforts of clinical practitioners and basic researchers.

\section{References}

[1] Dai, L.J., Li, H.Y., Guan, L.X., Ritchie, G. and Zhou, J.X. (2009) The Therapeutic Potential of Bone Marrow-Derived Mesenchymal Stem Cells on Hepatic Cirrhosis. Stem Cell Research, 2, 16-25.

[2] Eckersley-Maslin, M.A., Warner, F.J., Grzelak, C.A., McCaughan, G.W. and Shackel N.A. (2009) Bone Marrow Stem Cells and the Liver: Are They Relevant? Journal of Gastroenterology and Hepatology, 24, 1608-1616. https://doi.org/10.1111/j.1440-1746.2009.06004.x

[3] Mohamadnejad, M., Namiri, M., Bagheri, M., et al. (2007) Phase 1 Human Trial of Autologous Bone Marrow-Hematopoietic Stem Cell Transplantation in Patients with Decompensated Cirrhosis. World Journal of Gastroenterology, 13, 3359-3363. https://doi.org/10.3748/wjg.v13.i24.3359

[4] Nikeghbalian, S., Pournasr, B., Aghdami, N., et al. (2011) Autologous Transplantation of Bone Marrow-Derived Mononuclear and CD133(+) Cells in Patients with Decompensated Cirrhosis. Archives of Iranian Medicine, 14, 12-17.

[5] Mohamadnejad, M., Alimoghaddam, K., Mohyeddin-Bonab, M., et al. (2007) Phase 1 Trial of Autologous Bone Marrow Mesenchymal Stem Cell Transplantation in Patients with Decompensated Liver Cirrhosis. Archives of Iranian Medicine, 10, 459466.

[6] Caly, W.R., Strauss, E., Carrilho, F.J., Laudanna, A.A. (2003) Different Degrees of Malnutrition and Immunological Alterations According to the Aetiology of Cirrhosis: A Prospective and Sequential Study. Nutrition Journal, 2, 10. https://doi.org/10.1186/1475-2891-2-10

[7] Liu, Q. (2009) Role of Cytokines in the Pathophysiology of Acute-on-Chronic Liver Failure. Blood Purification, 28, 331-341. https://doi.org/10.1159/000232940 
[8] Saito, T., Okumoto, K., Haga, H., et al. (2011) Potential Therapeutic Application of Intravenous Autologous Bone Marrow Infusion in Patients with Alcoholic Liver Cirrhosis. Stem Cells and Development, 20, 1503-1510. https://doi.org/10.1089/scd.2011.0074

[9] Couto, B.G., Goldenberg, R.C., da Fonseca, L.M., et al. (2011) Bone Marrow Mononuclear Cell Therapy for Patients with Cirrhosis: A PHASE 1 Study. Liver International, 31, 391-400. https://doi.org/10.1111/j.1478-3231.2010.02424.x

[10] Jang, Y.O., Kim, Y.J., Baik, S.K., et al. (2014) Histological Improvement Following Administration of Autologous Bone Marrow-Derived Mesenchymal Stem Cells for Alcoholic Cirrhosis: A Pilot Study. Liver International, 34, 33-41. https://doi.org/10.1111/liv.12218

[11] Li, W.Y., Jiang, Y.F., Jin, Q.L., et al. (2010) Immunologic Characterization of Posthepatitis Cirrhosis Caused by HBV and HCV Infection. Journal of Biomedicine and Biotechnology, 2010, Article ID: 138237. https://doi.org/10.1155/2010/138237

[12] Salama, H., Zekri, A.R., Bahnassy, A.A., et al. (2010) Autologous CD $34^{+}$and $\mathrm{CD}_{133^{+}}$Stem Cells Transplantation in Patients with End Stage Liver Disease. World Journal of Gastroenterology, 16, 5297-5305. https://doi.org/10.3748/wjg.v16.i42.5297

[13] Cai, D.C., Li, J., Zeng, Y., Li, Y.G. and Ren, H. (2007) A Study on the Anti-HBV Effect of Dendritic Cell from Human Umbilical Cord Blood. Chinese Journal of Hepatology, 15, 88-91.

[14] Xing, T., Li, L., Cao, H. and Huang, J. (2007) Altered Immune Function of Monocytes in Different Stages of Patients with Acute on Chronic Liver Failure. Clinical \& Experimental Immunology, 147, 184-188.

[15] Weng, W.Z., Chen, J.F., Mei, Y.Y., et al. (2013) Treatment Effect of Allogeneic Bone Marrow Mesenchymal Stem Cells Transplantation to Patients with Different Phase of HBV Acute-on-Chronic Liver Failure. Journal of Sun Yat-sen University (Medical Science), 34, 422-428. (In Chinese)

[16] Houlihan, D.D. and Newsome, P.N. (2008) Critical Review of Clinical Trials of Bone Marrow Stem Cells in Liver Disease. Gastroenterology, 135, 438-450. https://doi.org/10.1053/j.gastro.2008.05.040

[17] Liu, F., Liu, Z.D., Wu, N., et al. (2009) Transplanted Endothelial Progenitor Cells Ameliorate Carbon Tetrachloride-Induced Liver Cirrhosis in Rats. Liver Transplantation, 15, 1092-1100. https://doi.org/10.1002/lt.21845

[18] Chen, C., Zeng, L., Ding, S. and Xu, K. (2010) Adult Endothelial Progenitor Cells Retain Hematopoiesis Potential. Transplantation Proceedings, 42, 3745-3749. https://doi.org/10.1016/j.transproceed.2010.07.094

[19] Oyagi, S., Hirose, M., Kojima, M., et al. (2006) Therapeutic Effect of Transplanting HGF-Treated Bone Marrow Mesenchymal Cells into $\mathrm{CCl}_{4}$-Injured Rats. Journal of Hepatology, 44, 742-748. https://doi.org/10.1016/j.jhep.2005.10.026

[20] Mohsin, S., Shams, S., Ali Nasir, G., et al. (2011) Enhanced Hepatic Differentiation of Mesenchymal Stem Cells after Pretreatment with Injured Liver Tissue. Differentiation, 81, 42-48. https://doi.org/10.1016/j.diff.2010.08.005

[21] Li, T.Z., Kim, J.H., Cho, H.H., et al. (2010) Therapeutic Potential of Bone-MarrowDerived Mesenchymal Stem Cells Differentiated with Growth-Factor-Free Coculture Method in Liver-Injured Rats. Tissue Engineering Part A, 16, 2649-2659. https://doi.org/10.1089/ten.tea.2009.0814

[22] Stock, P., Briickner, S., Ebensing, S., et al. (2010) The Generation of Hepatocytes from Mesenchymal Stem Cells and Engraftment into Murine Liver. Nature Proto- 
cols, 5, 617-627. https://doi.org/10.1038/nprot.2010.7

[23] Zhou, W., Chen, P.F., Wu, X.L., Jiang, R. and Xu, Y.H. (2012) Effect of Bone Marrow Mesenchymal Stem Cells on Experimental Liver Fibrosis in Rats and Relevant Mechanism. Chinese Journal of Biologicals, 25, 176-180. (In Chinese)

[24] Zhan, Y., Wang, Y., Wei, L., et al. (2006) Differentiation of Hematopoietic stem Cells into Hepatocytes in Liver Fibrosis in Rats. Transplantation Proceedings, 38, 3082-3085. https://doi.org/10.1016/j.transproceed.2006.08.132

[25] Ma, J.X., Yang, L.P., He, Z.J. and Fang, C.H. (2008) Rat Bone Marrow Mesenchymal Stem Cells Induced Hepatocyte-Like Transplantation for Repairing Acute Hepatic Injury. Journal of Clinical Rehabilitative Tissue Engineering Research, 12, 40264030. (In Chinese)

[26] Hwang, S., Hong, H.N., Kim, H.S., et al. (2012) Hepatogenic Differentiation of Mesenchymal Stem Cells in a Rat Model of Thioacetamide-Induced Liver Cirrhosis. Cell Biology International, 36, 279-288. https://doi.org/10.1042/CBI20110325

[27] Hardjo, M., Miyazaki, M., Sakaguchi, M., et al. (2009) Suppression of Carbon Tetrachloride-Induced Liver Fibrosis by Transplantation of a Clonal Mesenchymal Stem Cell Line Derived from Rat Bone Marrow. Cell Transplantation, 18, 89-99. https://doi.org/10.3727/096368909788237140

[28] Piryaei, A., Valojerdi, M.R., Shahsavani, M. and Baharvand, H. (2011) Differentiation of Bone Marrow-Derived Mesenchymal Stem Cells into Hepatocyte-Like Cells on Nanofibers and Their Transplantation into a Carbon Tetrachloride-Induced Liver Fibrosis Model. Stem Cell Reviews and Reports, 7, 103-118. https://doi.org/10.1007/s12015-010-9126-5

[29] Caplan, A.I. and Dennis, J.E. (2006) Mesenchymal Stem Cells as Trophic Mediators. Journal of Cellular Biochemistry, 98, 1076-1084. https://doi.org/10.1002/jcb.20886

[30] Harris, V.K., Faroqui, R., Vyshkina, T. and Sadiq, S.A. (2012) Characterization of Autologous Mesenchymal Stem Cell-Derived Neural Progenitors as a Feasible Source of Stem Cells for Central Nervous System Applications in Multiple Sclerosis. Stem Cells Translational Medicine, 1, 536-547. https://doi.org/10.5966/sctm.2012-0015

[31] Abdel-Aziz, M., Abdel-Hamid, S., Wahdan, O., et al. (2012) Phase II Trial: Undifferentiated versus Differentiated Autologous Mesenchymal Stem Cells Transplantation in Egyptian Patients with HCV Induced Liver Cirrhosis. Stem Cell Reviews and Reports, 8, 972-981. https://doi.org/10.1007/s12015-011-9322-y

[32] Zhang, B., Inagaki, M., Jiang, B., et al. (2009) Effects of Bone Marrow and Hepatocyte Transplantation on Liver Injury. Journal of Surgical Research, 157, 71-80. https://doi.org/10.1016/j.jss.2008.12.013

[33] Zagoura, D.S., Roubelakis, M.G., Bitsika, V., et al. (2012) Therapeutic Potential of a Distinct Population of Human Amniotic Fluid Mesenchymal Stem Cells and Their Secreted Molecules in Mice with Acute Hepatic Failure. Gut, 61, 894-906. https://doi.org/10.1136/gutjnl-2011-300908

[34] Wang, S., Yao, P., Gong, L.J., et al. (2009) Effect of Autologous Bone Marrow Mononuclear Cells Transplantation on Serum Cholinesterase in Patients with Liver Cirrhosis of Different Child-Pugh Scores. Chinese Hepatology, 14, 189-193. (In Chinese)

[35] Wang, S., Yao, P., Gong, L.J., et al. (2010) Effect of Autologous Bone Marrow Mononuclear Cells Transplantation on Serum Albumin in Patients with Liver Cirrhosis. Chinese Journal of Gastroenterology and Hepatology, 19, 509-512.

[36] Luo, L.L., Mu, X.L., Heng, L.N. and Lu, X.H. (2008) Human Bone Marrow Mesen- 
chymal Stem Cells Differentiate into Hepatocyte-Like Cells Induced by Serum of Hepatic Injury in Vitro. China Practical Medicine, 3, 3-4. (In Chinese)

[37] Geng, Y.J. (2003) Molecular Mechanisms for Cardiovascular Stem Cell Apoptosis and Growth in the Hearts with Atherosclerotic Coronary Disease and Ischemic Heart Failure. Annals of the New York Academy of Sciences, 1010, 687-697. https://doi.org/10.1196/annals.1299.126

[38] Yang, P.F., Chen, F.R., Guo, J.B., et al. (2005) Therapeutic Effect of Hyperbaric Oxygen on Cirrhotic Patients with Portal Hypertension. Chinese Journal of Gastroenterology and Hepatology, 14, 293-295. (In Chinese)

[39] Yang, F., Qin, B. and Qin, F. (2011) Influence of Artificial Liver Support System on Bone Marrow Stem Cell Differentiation Factors in Patients with Chronic Severe Hepatitis B. Chinese Journal of Infectious Diseases, 29, 674-678. (In Chinese)

[40] Xu, H.Y., Shi, X.L., Chu, X.H. and Ding, Y.T. (2009) Effects on Swine Acute Liver Failure by Combined Therapy of Autologous Mesenchymal Stem Cell Transplantation and Medical Treatment. World Chinese Journal of Digestology, 17, 962-968. (In Chinese) https://doi.org/10.11569/wcjd.v17.i10.962

[41] Tang, X.P., Zheng, X.H. and Yang, X. (2002) Therapeutic Effect of Umbilical Cord Blood Transfusion Combined with Plasma Exchange on Chronic Severe Hepatitis. Bulletin of Hunan Medical University, 27, 323-325. (In Chinese)

[42] Sun, Y., Chi, B.R., Chen, L., Meng, X.W. and Kong, D.X. (2008) Study on Transplantation of Induced Bone Marrow Mesenchymal Stem Cells via Various Route for the Treatment of Chronic Liver Injury. Chinese Journal of Digestion, 28, 171-174. (In Chinese)

[43] Stutchfield, B.M., Forbes, S.J. and Wigmore, S.J. (2010) Prospects for Stem Cell Transplantation in the Treatment of Hepatic Disease. Liver Transplantation, 16, 827-836. https://doi.org/10.1002/lt.22083

[44] Lu, Q.P., Cao, B.Q. and Wei, W. (2011) Different Component of Bone Marrow Cell Transplantation via the Portal Vein Controlled Study of the Treatment of Hepatic Fibrosis in Rats. Chinese Journal of Clinicians, 5, 4992-4996. (In Chinese)

[45] Wei, Y., Nie, Y., Lai, J., Wan, Y.J. and Li, Y. (2009) Comparison of the Population Capacity of Hematopoietic and Mesenchymal Stem Cells in Experimental Colitis Rat Model. Transplantation, 88, 42-48.

[46] Zhou, H.C., Liu, L., Zhou, J. and Tian, J.L. (2011) Comparison the Percentage of Lymphocytes, Monocytes and CD34, CD38 Isoforms Cells between Bone Marrow and Umbilical Cord Blood Stem Cells in Treatment of Liver Cirrhosis. Chinese Journal of Clinicians, 5, 1731-1733. (In Chinese)

[47] Liu, Z.C. and Chang, T.M. (2009) Preliminary Study on Intrasplenic Implantation of Artificial Cell Bioencapsulated Stem Cells to Increase the Survival of 90\% Hepatectomized Rats. Artificial Cells, Blood Substitutes, and Biotechnology, 37, 53-55. https://doi.org/10.1080/10731190802663975

[48] Lan, L., Chen, Y., Sun, C., et al. (2008) Transplantation of Bone Marrow-Derived Hepatocyte Stem Cells Transduced with Adenovirus-Mediated IL-10 Gene Reverses Liver Fibrosis in Rats. Transplant International, 21, 581-592. https://doi.org/10.1111/j.1432-2277.2008.00652.x

[49] Alexanian, A.R., Kwok, W.M., Pravdic, D., Maiman, D.J. and Fehlings, M.G. (2010) Survival of Neurally Induced Mesenchymal Cells May Determine Degree of Motor Recovery in Injured Spinal Cord Rats. Restorative Neurology and Neuroscience, 28, 761-767.

[50] Ji, W.J., Qu, Q., Jin, Y., Zhao, L. and He, X.D. (2009) Ursodeoxycholic Acid Inhibits 
Hepatocyte-Like Cell Apoptosis by Down-Regulating the Expressions of Bax and Caspase-3. National Medical Journal of China, 89, 2997-3001.

[51] Liao, X., AnCheng, J.Y., Zhou, Q.J. and Liao, C. (2013) Therapeutic Effect of Autologous Bone Marrow-Derived Liver Stem Cells Transplantation in Hepatitis B Virus-Induced Liver Cirrhosis. Hepatogastroenterology, 60, 406-409.

[52] Rabani, V., Shahsavani, M., Gharavi, M., et al. (2010) Mesenchymal Stem Cell Infusion Therapy in a Carbon Tetrachloride-Induced Liver Fibrosis Model Affects Matrix Metalloproteinase Expression. Cell Biology International, 34, 601-605. https://doi.org/10.1042/CBI20090386

[53] Liu, L., Zhou, J., Huang, L.W., He, C.P. and Zhou, H.C. (2011) Clinical Efficacy of Lamivudine, Adefovir Dipivoxil Combined with Autologous Bone Marrow Stem Cell Transplantation in Treatment of Hepatitis B Patients with Decompensated Liver Cirrhosis. Chinese Journal of Gastroenterology and Hepatology, 20, 10921094. (In Chinese)

[54] Deng, X., Chen, Y.X., Zhang, X., et al. (2008) Hepatic Stellate Cells Modulate the Differentiation of Bone Marrow Mesenchymal Stem Cells into Hepatocyte-Like Cells. Journal of Cellular Physiology, 217, 138-144.

https://doi.org/10.1002/jcp.21481

[55] Di Bonzo, L.V., Ferrero, I., Cravanzola, C., et al. (2008) Human Mesenchymal Stem Cells as a Two-Edged Sword in Hepatic Regenerative Medicine: Engraftment and Hepatocyte Differentiation versus Profibrogenic Potential. Gut, 57, 223-231. https://doi.org/10.1136/gut.2006.111617

[56] Ong, S.Y., Dai, H. and Leong, K.W. (2006) Hepatic Differentiation Potential of Commercially Available Human Mesenchymal Stem Cells. Tissue Engineering, 12, 3477-3485. https://doi.org/10.1089/ten.2006.12.3477

[57] Quintanilha, L.F., Mannheimer, E.G., Carvalho, A.B., et al. (2008) Bone Marrow Cell Transplant Does Not Prevent or Reverse Murine Liver Cirrhosis. Cell Transplantation, 17, 943-953. https://doi.org/10.3727/096368908786576453

[58] Carvalho, A.B., Quintanilha, L.F., Dias, J.V., et al. (2008) Bone Marrow Multipotent Mesenchymal Stromal Cells Do Not Reduce Fibrosis or Improve Function in a Rat Model of Severe Chronic Liver Injury. Stem Cells, 26, 1307-1314. https://doi.org/10.1634/stemcells.2007-0941

[59] Pulavendran, S., Rose, C. and Mandal, A.B. (2011) Hepatocyte Growth Factor Incorporated Chitosan Nanoparticles Augment the Differentiation of Stem Cell into Hepatocytes for the Recovery of Liver Cirrhosis in Mice. Journal of Nanobiotechnology, 9, 15. https://doi.org/10.1186/1477-3155-9-15

[60] Pulavendran, S., Vignesh, J. and Rose, C. (2010) Differential Anti-Inflammatory and Anti-Fibrotic Activity of Transplanted Mesenchymal vs. Hematopoietic Stem Cells in Carbon Tetrachloride-Induced Liver Injury in Mice. International Immunopharmacology, 10, 513-519. https://doi.org/10.1016/j.intimp.2010.01.014

[61] Kakinuma, S., Tanaka, Y., Chinzei, R., et al. (2003) Human Umbilical Cord Blood as a Source of Transplantable Hepatic Progenitor Cells. Stem Cells, 21, 217-227. https://doi.org/10.1634/stemcells.21-2-217

[62] Piscaglia, A.C., Zocco, M.A., Di-Campli, C., et al. (2005) How Does Human Stem Cell Therapy Influence Gene Expression after Liver Injury? Microarray Evaluation on a Rat Model. Digestive and Liver Disease, 37, 952-963. https://doi.org/10.1016/j.dld.2005.06.012

[63] Henning, R.J., Aufman, J., Shariff, M., et al. (2010) Human Umbilical Cord Blood Mononuclear Cells Decrease Fibrosis and Increase Cardiac Function in Cardiomyopathy. Regenerative Medicine, 5, 45-54. https://doi.org/10.2217/rme.09.71 
[64] Elkhafif, N., El-Baz, H., Hammam, O., et al. (2011) CD133 ${ }^{+}$Human Umbilical Cord Blood Stem Cells Enhance Angiogenesis in Experimental Chronic Hepatic Fibrosis. APMIS, 119, 66-75. https://doi.org/10.1111/j.1600-0463.2010.02693.x

[65] Wulf-Goldenberg, A., Eckert, K. and Fichtner, I. (2011) Intrahepatically Transplanted Human Cord Blood Cells Reduce SW480 Tumor Growth in the Presence of Bispecific EpCAM/CD3 Antibody. Cytotherapy, 13, 108-113. https://doi.org/10.3109/14653249.2010.515577

[66] Wang, Z.Y., Zhu, Y.J., Wang, Z. and Du, B. (2012) Therapeutic Effect of Umbilical Cord Blood Stem Cells Transplantation for Rat Model of Decompensated Cirrhosis. National Medical Frontiers of China, 7, 4-6. (In Chinese)

[67] Mehanna, R.A., Habachy, N.M., Sharara, G.M., et al. (2012) Transplantation of Human Umbilical Cord Blood Stem Cells in Rabbits' Fibrotic Liver. Journal of American Science, 8, 83-94.

[68] Bassiouny, A.R., Zaky, A.Z., Abdulmalek, S.A., et al. (2011) Modulation of AP-Endonuclease1 Levels Associated with Hepatic Cirrhosis in Rat Model Treated with Human Umbilical Cord Blood Mononuclear Stem Cells. International Journal of Clinical and Experimental Pathology, 4, 692-707.

[69] Sáez-Lara, M.J., Frecha, C., Martin, F., et al. (2006) Transplantation of Human CD34 ${ }^{+}$Stem Cells from Umbilical Cord Blood to Rats with Thioacetamide-Induced Liver Cirrhosis. Xenotransplantation, 13, 529-535. https://doi.org/10.1111/j.1399-3089.2006.00344.x

[70] Álvarez-Mercado, A.I., García-Mediavilla, M.V., Sánchez-Campos, S., et al. (2009) Deleterious Effect of Human Umbilical Cord Blood Mononuclear Cell Transplantation on Thioacetamide-Induced Chronic Liver Damage in Rats. Cell Transplantation, 18, 1069-1079. https://doi.org/10.3727/096368909X12483162197088

[71] Yang, H.L., Yu, F.T., Li, G.J., et al. (2010) Umbilical Cord Blood Stem Cell Transplantation for 86 Patients with Decompensated Cirrhosis. Shandong Medical Journal, 50, 16-17. (In Chinese)

[72] Zhang, L.X., Xing, L.H., Zhang, L.L., et al. (2010) Umbilical Cord Blood Stem Cell Transplantation in Treatment of Decompeusated Cirrhosis: A Preliminary Clinical Observation. Chinese General Practice, 13, 2680-2682. (In Chinese)

[73] Hu, X.X., Chen, H.O., Qian, L., et al. (2011) Umbilical Cord Blood Stem Cell Transplantation via Hepatic Artery in Treatment of Liver Cirrhosis. Chinese Journal of General Practitioners, 10, 58-60. (In Chinese)

[74] Xu, S. and Wang F. (2011) Umbilical Cord Blood Stem Cell Transplantation in Treatment of Decompensated Cirrhosis Complicated with Hepatic Diabetes: A Case Report. Journal of Clinical Hepatology, 14, 306-311. (In Chinese)

[75] Li, Q. and Li, C.H. (2010) Perioperative Nursing for a Patient with Wilson Disease in Unrelated Cord Blood Stem Cell Transplantation. Chongqing Medicine, 39, 3448-3449. (In Chinese)

[76] Li, C.Y., Zhao, J.L., Zhang, L., et al. (2010) Efficacy Comparison between Umbilical Cord Blood and Autologous Peripheral Blood Stem Cell Transplantation in the Treatment of Ascitic Cirrhosis. Chinese Journal of Blood Transfusion, 23, 182-184. (In Chinese)

[77] Sakuragawa, N., Enosawa, S., Ishii, T., et al. (2000) Human Amniotic Epithelial Cells Are Promising Transgene Carriers for Allogeneic cell Transplantation into Liver. Journal of Human Genetics, 45, 171-176. https://doi.org/10.1007/s100380050205

[78] Davila, J.C., Cezar, G.G., Thiede, M., et al. (2004) Use and Application of Stem Cells 
in Toxicology. Toxicological Sciences, 79, 214-223.

https://doi.org/10.1093/toxsci/kfh100

[79] Takashima, S., Ise, H., Zhao, P., Akaike, T. and Nikaido, T. (2004) Human Amniotic Epithelial Cells Possess Hepatocyte-Like Characteristics and Functions. Cell Structure and Function, 29, 73-84. https://doi.org/10.1247/csf.29.73

[80] Marongiu, F., Gramignoli, R., Dorko, K., et al. (2011) Hepatic Differentiation of Amniotic Epithelial Cells. Hepatology, 53, 1719-1729. https://doi.org/10.1002/hep.24255

[81] Moritoki, Y., Ueno, Y., Kanno, N., et al. (2007) Amniotic Epithelial Cell-Derived Cholangiocytes in Experimental Cholestatic Ductal Hyperplasia. Hepatology Research, 37, 286-294. https://doi.org/10.1111/j.1872-034X.2007.00049.x

[82] Miki, T. (2011) Amnion-Derived Stem Cells: In Quest of Clinical Applications. Stem Cell Research \& Therapy, 2, 25. https://doi.org/10.1186/scrt66

[83] Tee, J.Y., Vaghjiani, V., Liu, Y.H., et al. (2013) Immunogenicity and Immunomodulatory Properties of Hepatocyte-Like Cells Derived from Human Amniotic Epithelial Cells. Current Stem Cell Research \& Therapy, 8, 91-99.

https://doi.org/10.2174/1574888X11308010011

[84] Manuelpillai, U., Moodley, Y., Borlongan, C.V. and Parolini, O. (2011) Amniotic Membrane and Amniotic Cells: Potential Therapeutic Tools to Combat Tissue Inflammation and Fibrosis? Placenta, 32, S320-S325. https://doi.org/10.1016/j.placenta.2011.04.010

[85] Hong, S.B., Seo, M.S., Park, S.B., et al. (2012) Therapeutic Effects of Human Amniotic Epithelial Stem Cells in Niemann-Pick Type C1 Mice. Cytotherapy, 14, 630638. https://doi.org/10.3109/14653249.2012.663485

[86] Sant'Anna, L.B., Cargnoni, A., Ressel, L., Vanosi, G. and Parolini, O. (2011) Amniotic Membrane Application Reduces Liver Fibrosis in a Bile Duct Ligation Rat Model. Cell Transplantation, 20, 441-453. https://doi.org/10.3727/096368910X522252

[87] Manuelpillai, U., Tchongue, J., Lourensz, D., et al. (2010) Transplantation of Human Amnion Epithelial Cells Reduces Hepatic Fibrosis in Immunocompetent $\mathrm{CCl}_{4}$-Treated Mice. Cell Transplantation, 19, 1157-1168. https://doi.org/10.3727/096368910X504496 


\section{Abbreviations}

UCBSCs: human umbilical cord blood stem cells

hAECs: human amniotic epithelial cells

HSCs: Hematopoietic stem cells

MSCs: mesenchymal stem cells

ACLF: acute-on-chronic liver failure

BMSCs: bone marrow mesenchymal stem cells

ASCs: adipose-derived mesenchymal stem cells

EPCs: endothelial progenitor cells

ALB: serum albumin levels

HGF: hepatocyte growth factor

FGF: fibroblast growth factors

IL-10: interleukin-10

EGF: epidermal growth factor

BDLSCs: bone marrow-derived liver stem cells

UDCA: ursodeoxycholic acid

BMNCs: bone marrow mononuclear cells

UCBMNCs: umbilical cord blood mononuclear cells

PBSCs: peripheral blood stem cells

Submit or recommend next manuscript to SCIRP and we will provide best service for you:

Accepting pre-submission inquiries through Email, Facebook, LinkedIn, Twitter, etc. A wide selection of journals (inclusive of 9 subjects, more than 200 journals)

Providing 24-hour high-quality service

User-friendly online submission system

Fair and swift peer-review system

Efficient typesetting and proofreading procedure

Display of the result of downloads and visits, as well as the number of cited articles

Maximum dissemination of your research work

Submit your manuscript at: http://papersubmission.scirp.org/

Or contactym@scirp.org 